\title{
Editorial
}

\section{Liebe Leserinnen, liebe Leser,}

wir gratulieren Herr Prof. Dr. em. Friedhelm Schilling, einem bedeutsamen Fachvertreter und Wegbereiter der Motologie, zum 80. Geburtstag. Wir bedanken uns bei Sandra Mirbek und Frank Francesco Birk, die diesen Geburtstag zum Anlass genommen haben, unter dem Fokus »Motologie gestern und heute: Ein Rückblick« ein Interview mit Friedhelm Schilling zu führen und in diesem Heft der »motorik« zu veröffentlichen.

Zu einem aktuellen motologischen Fachdiskurs führt Benajir Wolf mit ihrer These "Körperpsychotherapie und Motologie sind in der klinischen Praxis kaum zu unterscheiden«. Diese These leitet ihren Beitrag über den Vergleich von Arbeitsmodi und -haltungen dieser beiden Ansätzen ein. Sie kommt dabei als Expertin zu Wort, die in beiden Fachdiskursen zu Hause ist. Sie ermöglicht einen Blick durch »zwei Türen, die in den selben Raum führen«.

Die Förderung sozialer Kompetenzen gilt in der psychomotorischen Entwicklungsförderung und -begleitung als eine Zielsetzung in Richtung Handlungskompetenz. Auch im schulischen Kontext wird die Förderung sozialer Kompetenzen im Rahmen von sogenannten Sozialtrainings eingesetzt. Welchen Anteil und welche Bedeutung Bewegung in diesen Sozialtrainings einnimmt, wird in dem empirischen Artikel von Karina Teschke, Caterina Schäfer und Anna Südkamp ausgewertet.

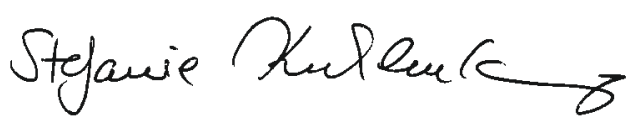

Stefanie Kuhlenkamp
Einen Beitrag zur Diskussion psychomotorischer Förderangebote in der Kita können die Ergebnisse der Wirksamkeitsstudie zu motorischen Fördermaßnahmen im Kindergartenalter von Heinz Krombholz liefern. Er evaluiert hierzu verschiedene Interventionsstudien in Münchener Kindertagesstätten.

Einem aktuellen Thema widmet sich Marianne Herzog, indem sie für die Rubrik Wissen kompakt das Thema »Traumapädagogik"

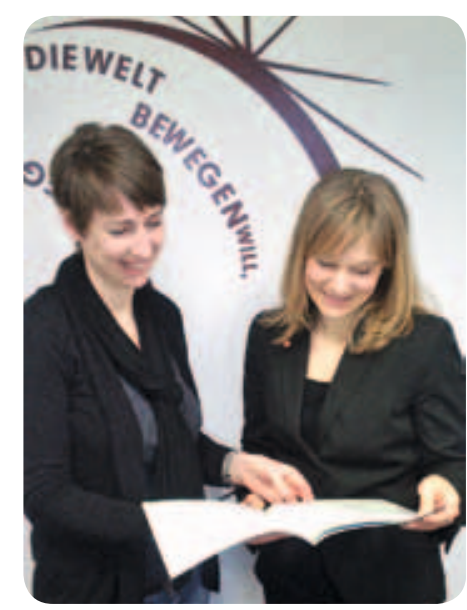

v. l.n. r.: Britta Gebhard, Stefanie Kuhlenkamp aufarbeitet und einen Bezug zur psychomotorischen Förderung herstellt. Das im Beitrag angesprochene Bilderbuch »Lily, Ben und Omid «, welches in kindgerechter Sprache und Illustration von der Traumabewältigung handelt, wird im Servicebereich vorgestellt. Es bietet eine gute Möglichkeit des aktiven Einbezugs für die Arbeit mit Kindern, die traumatische Erfahrungen machen mussten.

Kinderbuchfiguren als stärkende Vorbilder für Kinder in der psychomotorischen Arbeit stellt Gisela Schlesinger in Theorie und Praxis im Fachforum und im Praxistipp vor.

Wir wünschen Ihnen eine anregende Herbst-Lektüre. Mit freundlichen Grüßen aus Dortmund und Nordhausen

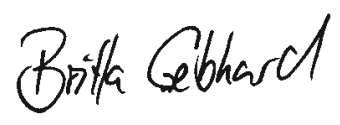

Britta Gebhard 